\title{
Metabonomics Research of the Four Properties in Traditional Chinese Medicine Based on UPLC-QTOF-MS System
}

\author{
Haixue Kuang, Yanyan Wang, Qiuhong Wang, \\ Bingyou Yang and Yonggang Xia \\ Key Laboratory of Chinese Materia Medica (Heilongjiang University of Chinese Medicine), \\ Ministry of Education, Harbin, \\ China
}

\section{Introduction}

TCM has long been practised as an empirical system and retrieved tens of millions of lives from historically to currently [1]. It is considered as an ancient and classical paradigm of systems biology. In TCM, diagnosis and medication are based on "Syndrome" ("ZHENG" in Chinese Mandarin), which can be regarded as a profile of symptom combination, or clinical phenotypes, such as Cold or Hot Syndrome, and "Hot medication curing Cold Syndrome and Cold medication curing Hot Syndrome" is a standard therapeutic guide line. This classical systems medicine at the macro level has been validated and developed by its repeated clinical practice for thousands of years [2]. Hot and Cold medication are the four properties of Chinese medicinal herbs, precisely including cold, hot, warm and cool, which are also called the four natures or "four xing" in TCM. Cold-cool and warm-hot are two completely opposite categories of natures, whereas cold and cool or hot and warm differ in the degree. Chinese medicinal herbs with cold and cool nature can clear away heat, purge fire and eliminate toxic materials, which are mainly used for heat-syndrome; while with hot and warm nature have the actions of expelling cold, which are mainly used for coldsyndrome. The four natures are summarized mainly from the body's response. On the base of syndrome differentiation theory, only distinguish heat or cold nature of disease, and have a good understanding of the cold or hot property of TCM, so selectively apply corresponding medicinal herbs that you could achieve the desired effect.

Herbal medicine has attracted much attention as a means of alternative therapy along with the orthodox medical system [3, 4]. In recent 30 years more and more reports on the four natures of TCM have appeared in the literature. To date several aspects of research such as the characteristics of thermodynamics, the changes of nervous system and the endocrine glands, energy metabolism, the systems biology analysis include genome, transcriptome, proteome, and metabolome are all supposed to explored the macro and micro framework on the four properties, among which metabonomics is the most novel tool [5]. It is a rapidly growing area of scientific research, which has been widely used in disease diagnosis, biomarker discovery, and research into the disease mechanisms [6-10]. Metabonomics aims at comparing the pattern of endogenous metabolites under defined temporal conditions as 
comprehensively as possible. In the past few years, widely used analytical techniques in metabonomics were nuclear magnetic resonance spectroscopy (NMR) [11], mass spectrometry in combination with gas chromatography (GC-MS) [12], capillary electrophoresis (CE-MS) [13] and especially liquid chromatography (LC-MS) [14]. Because of the advantages of robust operation and usability, separation with a reversed-phase column is a routine in LC-MS based metabonomic analysis. Advances in mass spectrometric techniques, particularly when linked to liquid chromatography, have resulted in the development of robust methods for low molecular mass organic molecules in biological matrices $[15,16]$. Indeed in a preliminary study the potential for LC-MS to detect differences in the composition of urine from control and dosed animals in a properties study of TCM has been demonstrated. Here we describe preliminary studies on the variety of the endogenous metabolite of different herbs.

Metabonomic strategies produces complex data sets, and therefore, the uses of appropriate multivariate statistical and visualization tools are mandatory keys that include efficient and robust methods to model, analyze, and interpret the complex chemical and biological data [21]. In metabonomics studies chemometrics analysis techniques including PCA and OPLSDA are often used to classify the samples[17]. Loading plots and Variable importance in the Project (VIP) value are commonly used for biomarker selection. Besides, the S-plot also plays an important role in screening for statistically significant compounds. It is a scatter plot which combines the covariance and correlation in loading results.

In this work, we employed a metabonomics strategy based on UPLC-QTOF-MS to discriminate the global urine profiles, and PCA was performed to detect the perturbation metabolites as many as possible. Furthermore, the potential biomarkers were screened out, which might be the target components in the future pathogenesis research, as well as predicted model was builded up using OPLS-DA. One of the intentions is to discuss whether this model is suitable for other herbs. The other purpose is to find potential criterion to evaluate the properties in TCM.

\section{Experimental}

\subsection{Reagents and materials}

Acetonitrile (HPLC grade) was purchased from Fisher Scientific (Loughborough, UK); formic acid (HPLC grade) used as mobile phase additives (each of purity $\geq 99 \%$ ), were supplied by Sigma-Aldrich (MO, USA); the distilled water was purified by a Milli-Q system (Millipore, MA, USA); Pentobarbital sodium was purchased from the Shanghai Chemical Agent Company of China Medicine Clique (Shanghai, China); Leucine-enkephalin was obtained from Sigma-Aldrich (MO, USA).

The six crude drugs, Rhizoma Coptidis, Radix Scutellariae, Cortex Phellodendri, Radix Aconiti Lateralis Preparata, Rhizoma Zingiberis Pricklyash Peel and Flos Datura were purchased from the Harbin Tongrentang Drugstore and were authenticated by Professor Zhenyue Wang of the Department of Pharmacognosy, Heilongjiang University of Chinese Medicine. The crude drugs were refluxed extraction with distilled water two times for an hour each time. The extracts of the six herbs were filtered and concentrated, their concentrations were shown in Table 1 , and stored in $4^{\circ} \mathrm{C}$ for animal experimental usage.

\subsection{Animals and dosing}

Male Sprague-Dawley rats ( $n=8$ per group), approx. $220 \mathrm{~g}$ in weight, were allowed to acclimatize in metabolism cages for 5 days prior to treatment. Food and water was provided 
ad libitum. The whole procedure of administration for each group was shown in Table 1 . Urine was collected every other day (7:00 pm-7:00 am) from metabolism cages at ambient temperature throughout the whole procedure and centrifuged at $12,000 \mathrm{rpm}$ at $4^{\circ} \mathrm{C}$ for 10 $\mathrm{min}$, and then the supernatants were aliquot into eppendorf tubes with $1.5 \mathrm{~mL}$ urine in each stored at $-20^{\circ} \mathrm{C}$ until analysis. In the exploratory study, in order to improve the matching rate of subjects, all groups were male. And there were no diet while drink free in the sample collection.

\begin{tabular}{llll}
\hline No. & Groups & $\begin{array}{l}\text { Concentrations of } \\
\text { crude drugs }(\mathrm{g} / \mathrm{ml})\end{array}$ & $\begin{array}{l}\text { Administration } \\
\text { dosage }(\mathrm{g} / \mathrm{kg})\end{array}$ \\
\hline 1 & control & - & 7.0 \\
2 & Rhizoma Coptidis & 0.35 & 6.0 \\
3 & Radix Scutellariae & 0.30 & 8.4 \\
4 & Cortex Phellodendri & 0.42 & 10.5 \\
5 & $\begin{array}{l}\text { Radix Aconiti Lateralis } \\
6\end{array}$ & 0.525 & 8.4 \\
7 & $\begin{array}{l}\text { Preparata } \\
\text { Rhizoma Zingiberis }\end{array}$ & 0.42 & 4.0 \\
\hline
\end{tabular}

Table 1 . The whole procedure of administration for each group, mean $\pm S D, n=8$.

\subsection{UPLC-QTOF-MS}

\subsubsection{Reversed-phase liquid chromatography}

Urine samples were centrifuged again at $12,000 \mathrm{rpm}$ for $10 \mathrm{~min}$ at $4^{\circ} \mathrm{C}$, the supernatants were transferred to autosampler vials for analysis. The autosampler was maintained at a temperature of $4^{\circ} \mathrm{C}$ for the duration of the analysis. The UPLC-MS analysis was performed on a Waters ACQUITYTM UPLC system (Waters Corporation, MA, USA). Separation was achieved on an ACQUITY UPLCTM BEH $\mathrm{C}_{18}$ column $(50 \mathrm{~mm} \times 2.1 \mathrm{~mm}$, i.d., $1.7 \mu \mathrm{m})$ maintained at $40{ }^{\circ} \mathrm{C}$. The column was eluted with a linear gradient of $2-40 \% \mathrm{~B}$ over $0-8.0$ min, 40-98 \% B over 8.0-10.0 $\mathrm{min}, 98-2 \%$ B over 10.0-12.0 min and kept at 2\% B for $2 \mathrm{~min}$, the composition was held at a flow rate of $600 \mu \mathrm{l} / \mathrm{min}$, where mobile phase A consisted of $0.1 \%$ formic acid in demonized water and mobile phase B consisted of $0.1 \%$ formic acid in acetonitrile. The injection volume was $2 \mu \mathrm{l}$ and the gradient duration was $14 \mathrm{~min}$. A blank was analyzed between every five samples to wash the column.

\subsubsection{Mass spectrometry}

A Waters Xevo quadrupole time-of-flight Mass Spectrometer (Manchester, UK) equipped with an electrospray ionization (ESI) source was used to collect metabolic profiling. Mass spectrometry was operated in the positive ion mode, according to our preliminary experiments for determination of system, the optimal conditions were as follows: capillary voltage of $3.0 \mathrm{kV}$, cone voltage of $40 \mathrm{~V}$, source temperature of $120^{\circ} \mathrm{C}$ and desolvation temperature of $400^{\circ} \mathrm{C}$. Nitrogen was used as the desolvation and cone gas with the flow rate of 
800 and $50 \mathrm{~L} / \mathrm{h}$, respectively. The data acquisition rate was set to $0.2 \mathrm{~s}$ with a $0.02 \mathrm{~s}$ interscan delay. The scan range was from 100 to $1000 \mathrm{~m} / \mathrm{z}$. All analyses were acquired by using the lock spray to ensure accuracy and reproducibility, leucine-enkephalin was used as the lock mass at a concentration of $400 \mathrm{pg} / \mathrm{ml}$ in acetonitrile $(0.1 \%$ formic acid $): \mathrm{H}_{2} \mathrm{O}(0.1 \%$ formic acid $)$ for the positive ion mode $\left([\mathrm{M}+\mathrm{H}]^{+}=556.2771\right)$. Data were collected in pareto mode, the lock spray frequency was set at $1 \mathrm{~s}$ and the lock mass data were averaged over 10 scans for correction. A "purge-wash-purge" cycle was employed on the autosampler with $90 \%$ aqueous formic acid used for the wash solvent and $0.1 \%$ aqueous formic acid used as the purge solvent.

For the further identification of potential markers, a mass spectrometric data were collected in full scan auto mode from 0 to $14 \mathrm{~min}$ in positive ion mode. In the MS/MS experiments, the conditions were the same as above except the collision energy was set from 15 to $30 \mathrm{eV}$ for each analyte.

\subsection{Data processing and statistical analysis}

UPLC-MS data were analyzed with the MassLynx software version V4.1 (Waters Corporation, Milford, USA). Before multivariate statistical analysis, the data of each sample was normalized to total area to correct for the MS response shift from the first injection to the last injection due to the long duration, overnight or longer, of an LC-MS analysis in metabonomics studies. The main parameters were set as follows: retention time range 1-9 min, mass range $100-1000$, mass tolerance 0.05 , mass window 0.05 , retention time window 0.20, noise elimination level 6. the MarkerLynx Application Manager (Waters Corporation, Milford, USA) was used for the peak detection and the EZinfo software was used for PCA and OPLS-DA. Pareto scaling was used in all the models to avoid chemical noise. Potential biomarkers were selected according to Variable importance in the Project (VIP) value, the loading plot and the S-plot. For the identification of potential markers, the following databases have been used: HMDB (http://www.hmdb.ca/), METLIN (http://metlin.scripps.edu/), Massbank (http://www.massbank.jp), PubChem (http://ncbi.nim.nih.gov/) and KEGG (http://www.kegg.com/).

\section{Results}

\subsection{UPLC-QTOF-MS method development}

In this study, non-targeted analyses of Urine samples metabolic components were performed. As there was no specific group of target analyses, some generic settings had to be applied both to LC separation and MS detection during the method development, in order to obtain urine metabolic profiling containing as many compounds as possible. UPLC employs smaller stationary phase particle size column, generating high efficiency to the separation, which concurrently increases resolution and sensitivity. All urine metabolites were eluted in $14 \mathrm{~min}$ and full scan was set in the positive ion mode because it gave more information rich data than negative ion mode. For LC-MS-based metabonomics, the stability of analytical system is one of the most important factors to obtain the valid data. Extracted ion chromatographic peaks of seven ions (m/z 105.04, 154.02, 267.08, 271.08, 338.04, 340.03 and 675.13 in positive ion mode) were selected for method validation. Method repeatability was evaluated by five replicate analysis of a urine sample. The relative standard deviations (R.S.D.s\%) of peak areas and retention times were estimated to be $1.14-3.52 \%$ and 0.445 $1.36 \%$, respectively. The stability of sample was tested by analyzing a sample left at autosampler (maintained at $4{ }^{\circ} \mathrm{C}$ ) for $4,8,12$ and 24 h. The R.S.D.s\% of peak areas were from 
$4.58 \%$ to $7.54 \%$. These results demonstrated the excellent stability and reproducibility of chromatographic separation and mass measurement during the whole sequence [18].

\subsection{Chemometric analysis}

\subsubsection{Metabolic profiling analysis}

The positive ion base peak intensity (BPI) chromatograms of urine samples collected from representative rats for each of the different groups are presented in Fig. 1 Urine samples on the 30th day from each group were used for UPLC-MS analysis. Some differences could be visually noted among these chromatograms.
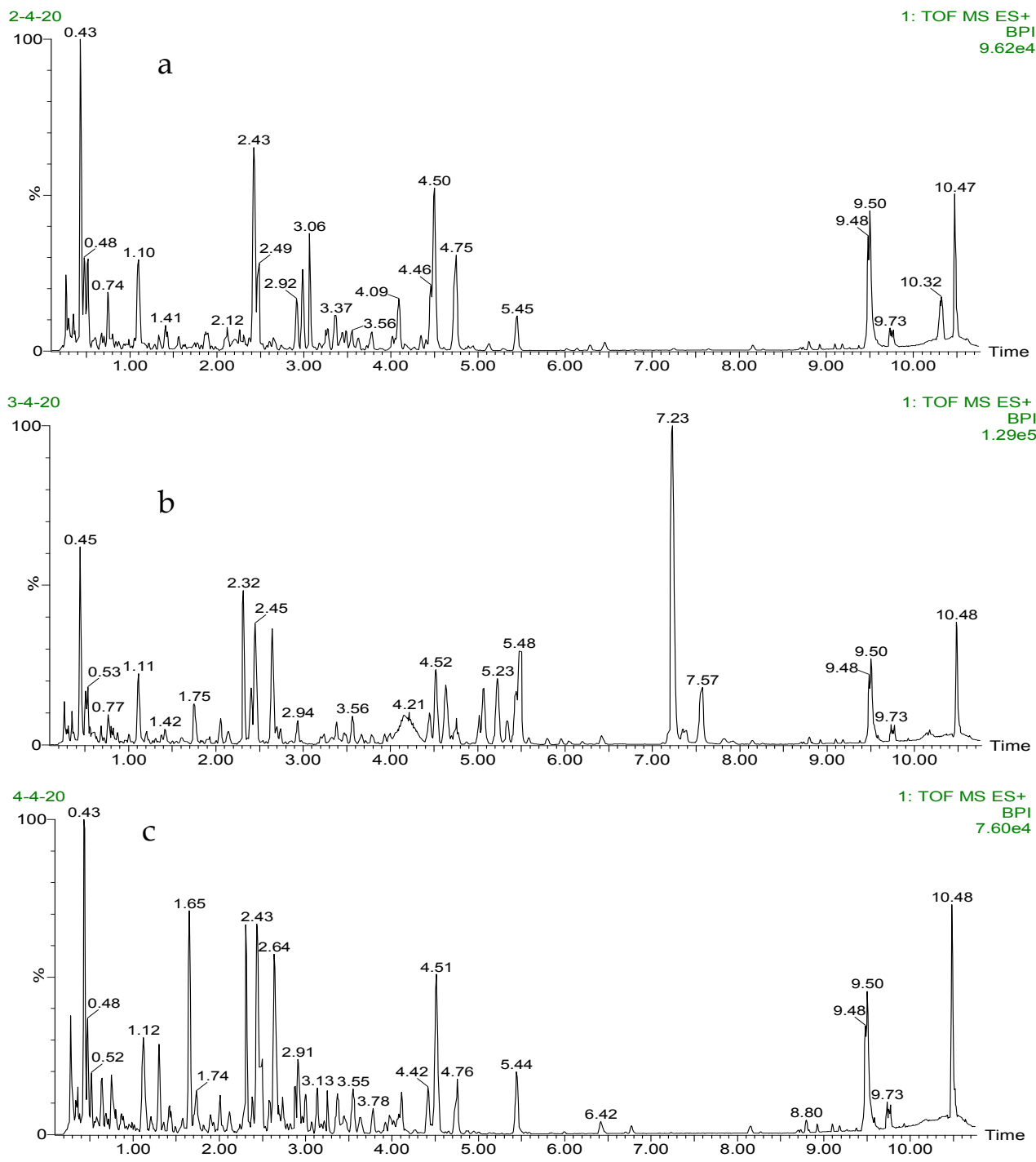

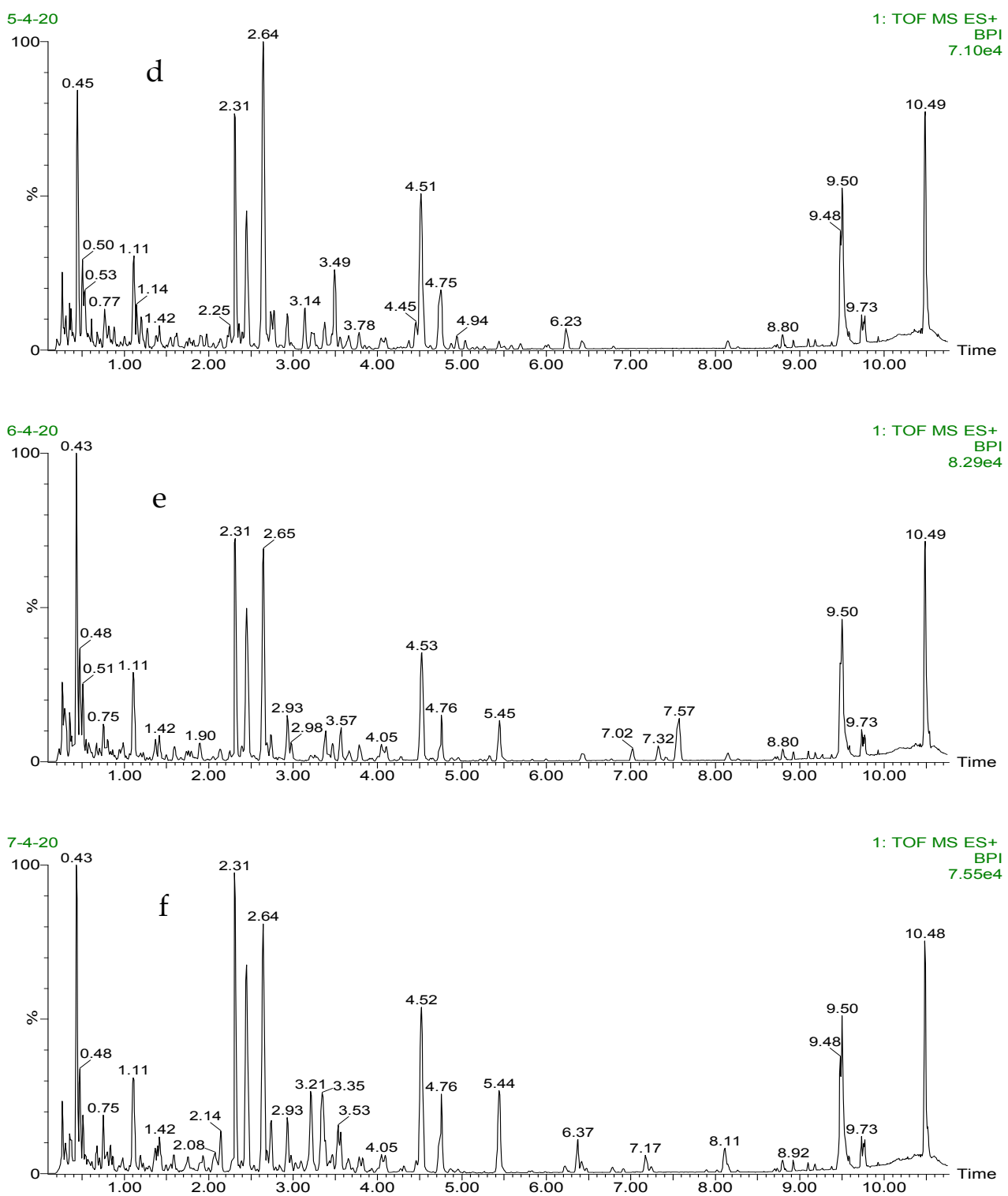

Fig. 1. BPI chromatograms of (a) Rhizoma Coptidis, (b) Radix Scutellariae, (c) Cortex Phellodendri, (d) Radix Aconiti Lateralis Preparata (e) Rhizoma Zingiberis (f) Rhizoma Zingiberis and Pricklyash Peel groups urine samples on the last day analyzed by UPLC-MS in positive ESI mode. 


\subsubsection{PCA analysis}

In order to clearly differentiate among groups, unsupervised pattern recognition PCA was used for analyzing the chromatographic data. PCA is a bilinear decomposition method that allows original data to be reduced to a few principal components while retaining the features that contribute most to the variance. It does not require any prior knowledge of class membership and was used here to detect any inherent trends within the data and to identify potential markers. The urinary data was subjected to EZinfo software, PCA score plots separated urine samples into different blocks, and samples with the same treatment were located on the same trajectory, indicating that treatments have greatly disturbed the normal urine metabolic profiles of rats (Fig. 2). Urine from the 2-4 groups were further from control group, suggesting the metabolic profiles have significantly changed as a result of Cold-cool medication administration. Rats in 5-7 groups were administrated with warm-hot medication for 24 consecutive days, the results were separated from the control group, demonstrating that the endogenous metabolic disorders occured after stimulus from warmhot medication, nevertheless, the perturbation of metabolic profiles are different from Cold medication group even with opposite trend. It was consistent with the clinical report that the four natures cold and hot are summarized mainly from the body's response after taking Chinese medicinal herbs are taken. Following the above data pre-treatment, PCA was employed in the first phase of chemometric analysis for positive data to evaluate urine sample clustering according to the character of the herbs variety, which is the most suitable for discrimination among the sample classes.

\subsubsection{OPLS-DA analysis}

In order to obtain better discrimination between different characteristic herbs and find the significant change of endogenous metabolites (i.e. potential biomarkers), an OPLS-DA model was constructed. OPLS-DA score plots separated urine samples of control group and Chinese medicinal herbs with cold nature groups as well as hot nature groups into two blocks, respectively, especially in the component P1 direction, and component P2 properly explained individual variation in each group (Fig. 3), which indicates biochemical changes happened in the urinary of male rats after the treatment of different property herbs. Loadings for component P1 indicated the content of each ion in the control and herbs groups; the $\mathrm{Y}+$ axis represented the herbs group; the $\mathrm{Y}$-axis represented the control group; the $\mathrm{X}$-axis represented the number of detected ions. To exhibit the responsibility of each ion for these variations more intuitively, S-plots and VIP-value plots were shown (Fig. 4). In the S-plot, most of the ions were clustered around the origin point; only a few of them scattered in the margin region, and just these few ions contributed to the clustering observed in the score plot and were also the differentiating metabolites. The VIP-value plot represents the value of each ion. The farther away from the origin, the higher the VIP value of the ions was. As illustrated in Fig. 3, Urine of the six herbs-treated groups were analyzed by OPLS-DA, the result of score plots indicating Cold and hot medication could separated well from each other, Rhizoma Coptidis, Radix Scutellariae and Cortex Phellodendri-treated groups have similar metabolic profiles were located the same quadrant, Similarly to Radix Aconiti Lateralis Preparata, Rhizoma Zingiberis and Pricklyash Peel-treated groups. The analysis of the chromatographic data identified the Cold and hot medication-treated rats based on the differences in their metabolic profiles, demonstrating that the classic herbs showed significant intervened effects on the normal rats, which was consistent with the theory of clinical medicine. 


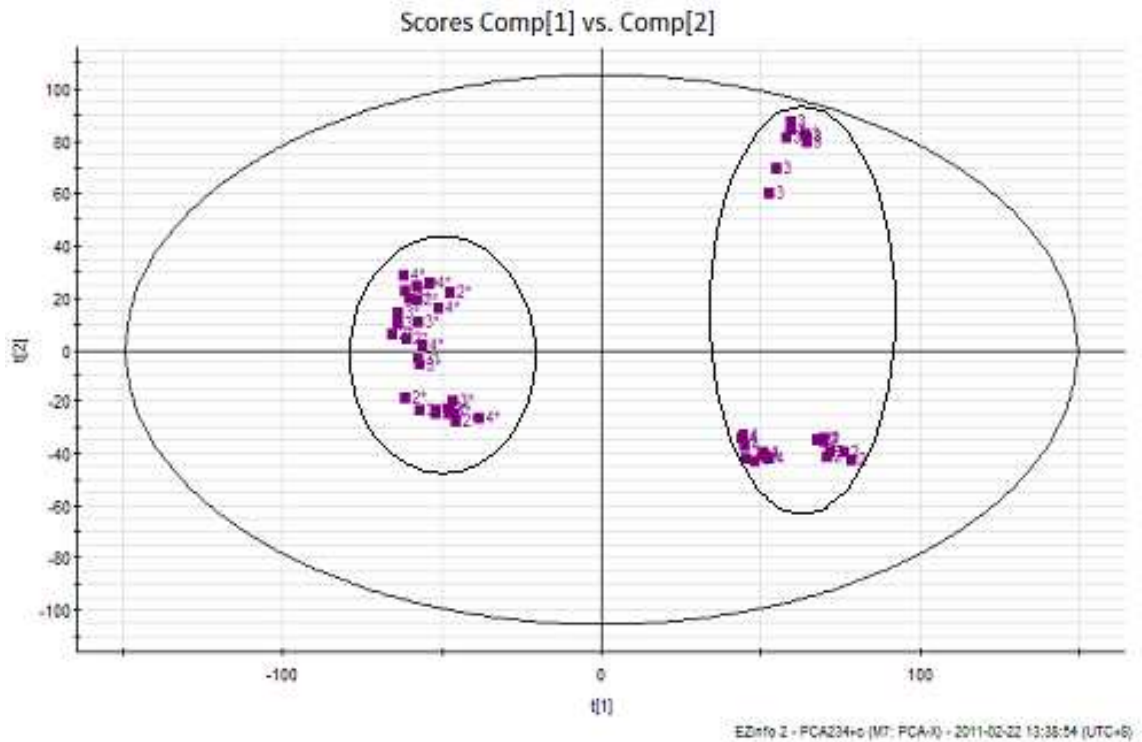

A

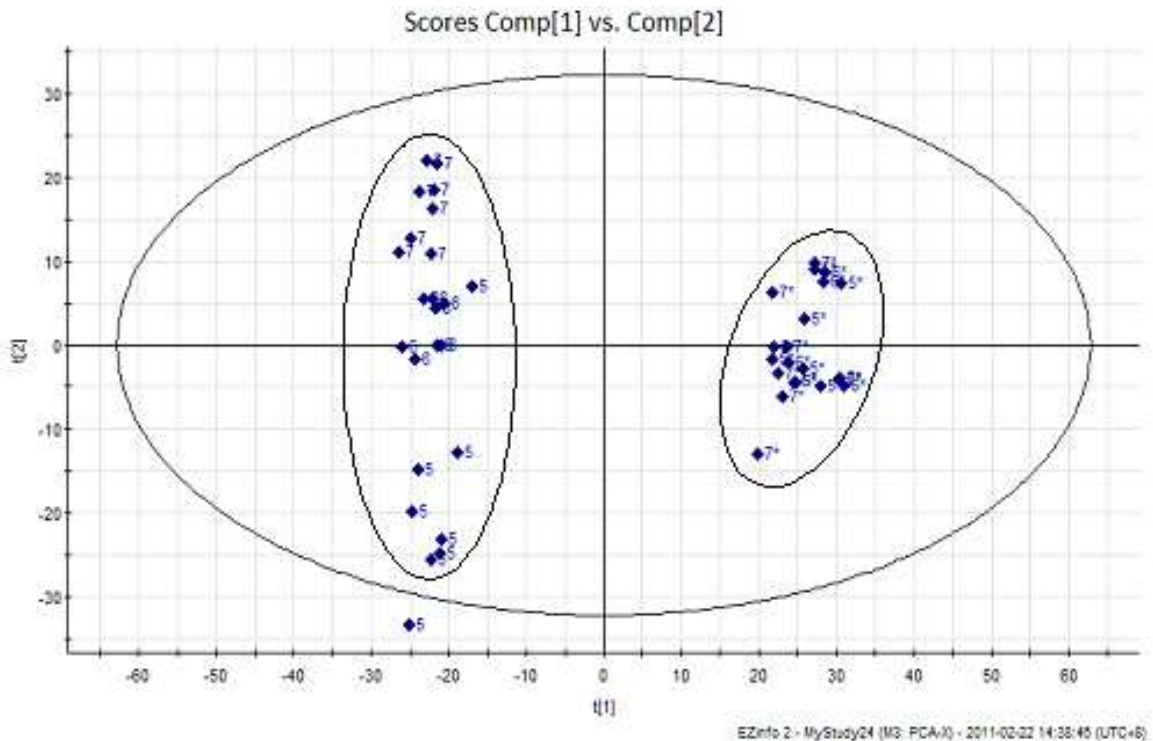

$\mathrm{B}$

Fig. 2. PCA scores plots derived from the UPLC-QTOF spectra of the urine in positive mode. (A) 2-4 groups originated from Rhizoma Coptidis, Radix Scutellariae and Cortex Phellodendri, belong to cold medication $2^{*}-4^{*}$ groups originated from control groups. (B) 5-7 groups originated from Radix Aconiti Lateralis Preparata, Rhizoma Zingiberis and Rhizoma Zingiberis and Pricklyash Peel groups, belong to hot medication groups and $5^{*}-7^{*}$ groups originated from control groups. 


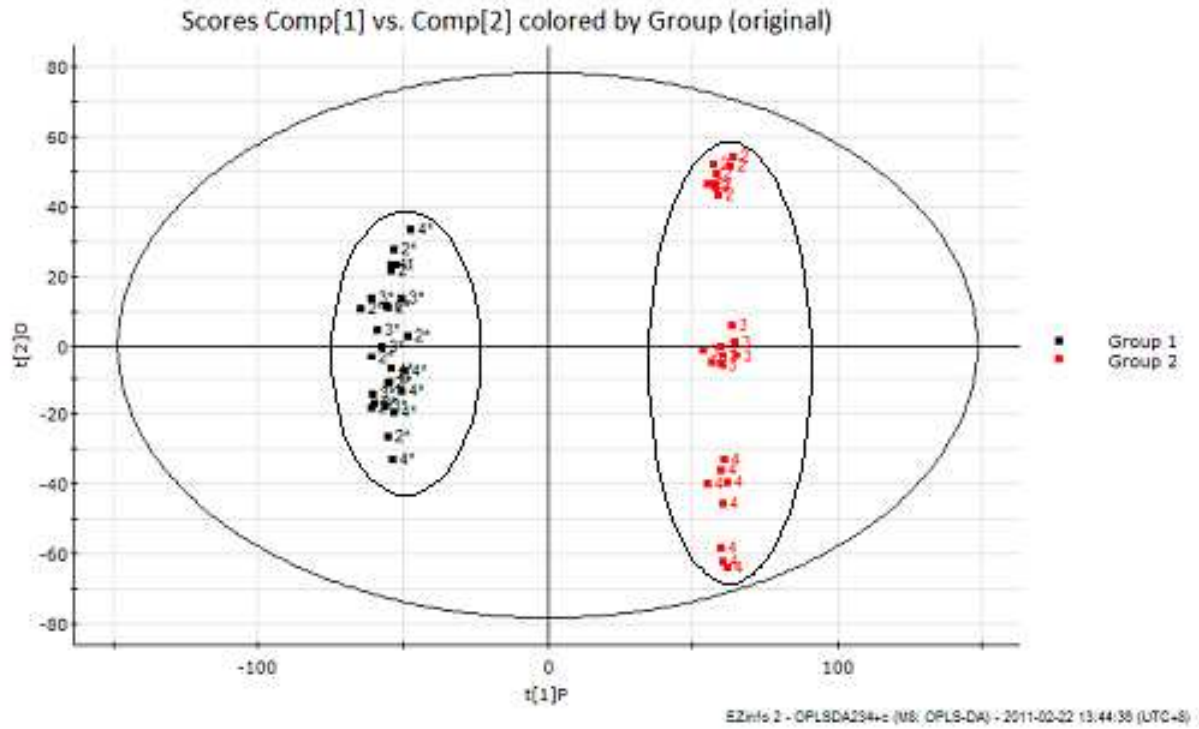

A

Scores Comp[1] vs. Comp[2] colored by Group (original)

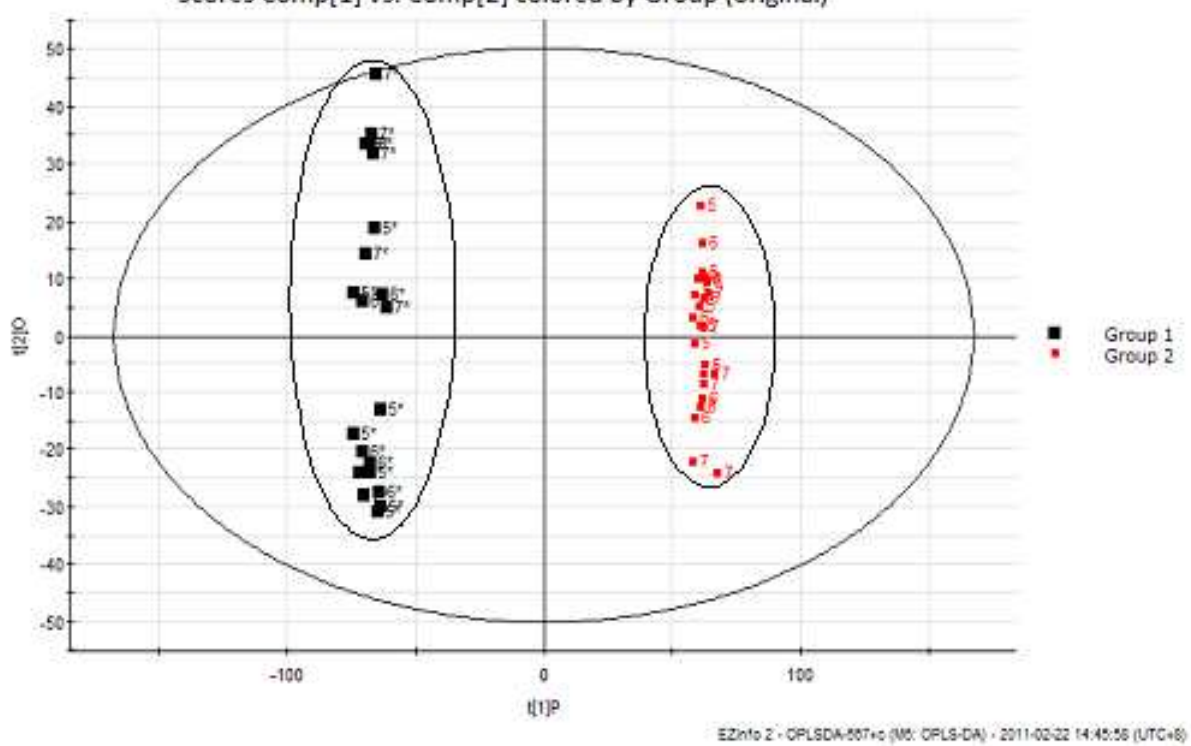




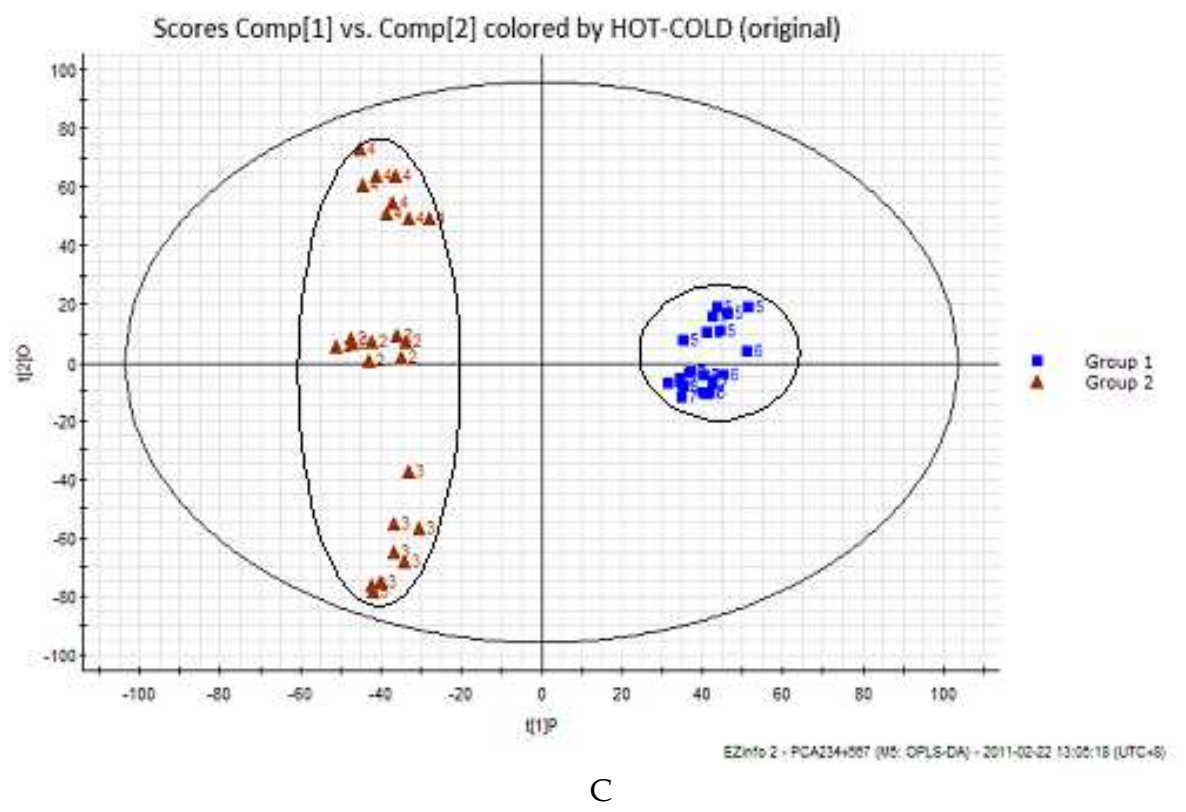

Fig. 3. OPLS-DA scores plots to classification the six groups of cold and hot medication. A, (घ) Group 1 is cold medication group and ( $\square$ ) group 2 is control group. B, (घ) Group 1 is hot medication group and ( $\square$ )group 2 is control group. $\mathbf{C},(\mathbf{\square})$ Group 1 is hot medication group and $(\mathbf{\Delta})$ group 2 is cold medication group.

For demonstration of the discrimination potential offered by the above data, OPLS-DA, a widely used supervised pattern recognition method capable of sample class prediction, was used to construct and validate a statistical model for traditional Chinese medicine classification. To estimate the predictive ability of our model, we used another herb-Flos Datura to cross-validation, and excellent separation among the TCM varieties obtained by OPLS-DA is shown in Fig. 5, which a hot medication belonging to the hot medication group, are presented in terms of recognition and prediction abilities. It represented the percentage of the samples correctly classified during model training and cross-validation, respectively, while the prediction ability was only qualitative rather than quantitative.

\subsubsection{Biomarker identification}

According to the VIP values of independent test (Table 2), these ions show significant differences between the controls and TCM groups. The same trend ions of three cold-cool or warm-hot medication groups were found, they may be biomarker candidates to reflect metabolic differences on the four properties of TCM. All the detected ions were arranged in descending order according to VIP values, and the highest VIP value was 20.75 in the positive mode. Combining the results of the OPLS analysis with the amount variation of ions in each group, 9 ions with VIP values exceeding two were selected preliminary identified in the Cold medication groups. At the same time, the highest VIP value was 12.73 in the positive mode, and 9 ions with VIP values exceeding two were selected and preliminary identified. The UPLC-MS segregation analysis platform provided the retention 


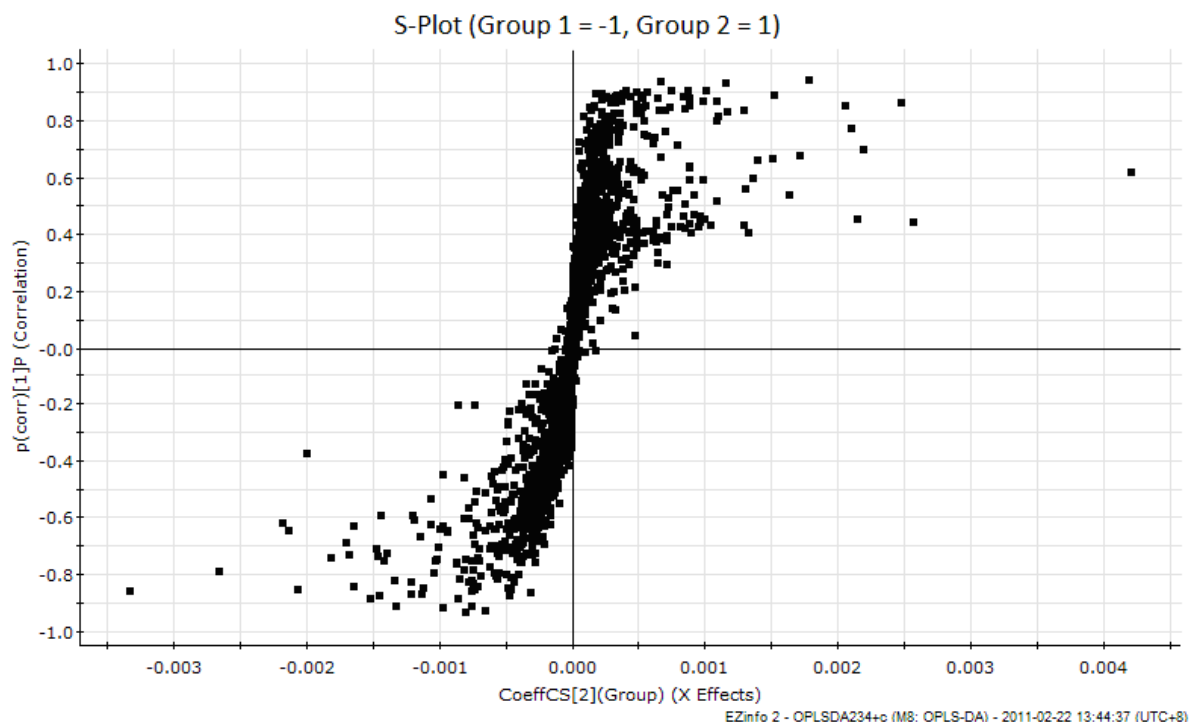

A

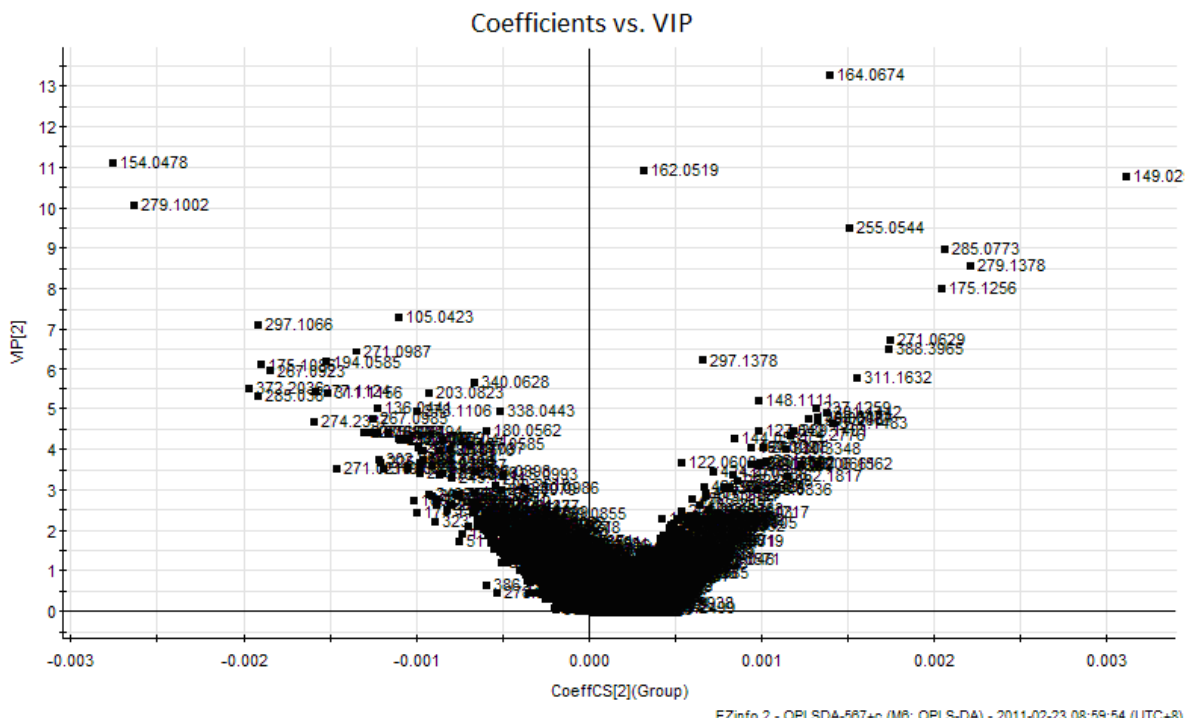

B

Fig. 4. S-plot and VIP values in positive ESI mode. A, S-plot of Cold medication group(Gruop 1 originated from cold medication, and group 2 originated from control ones). $\mathrm{B}$, VIP values plot of Hot medication group. 


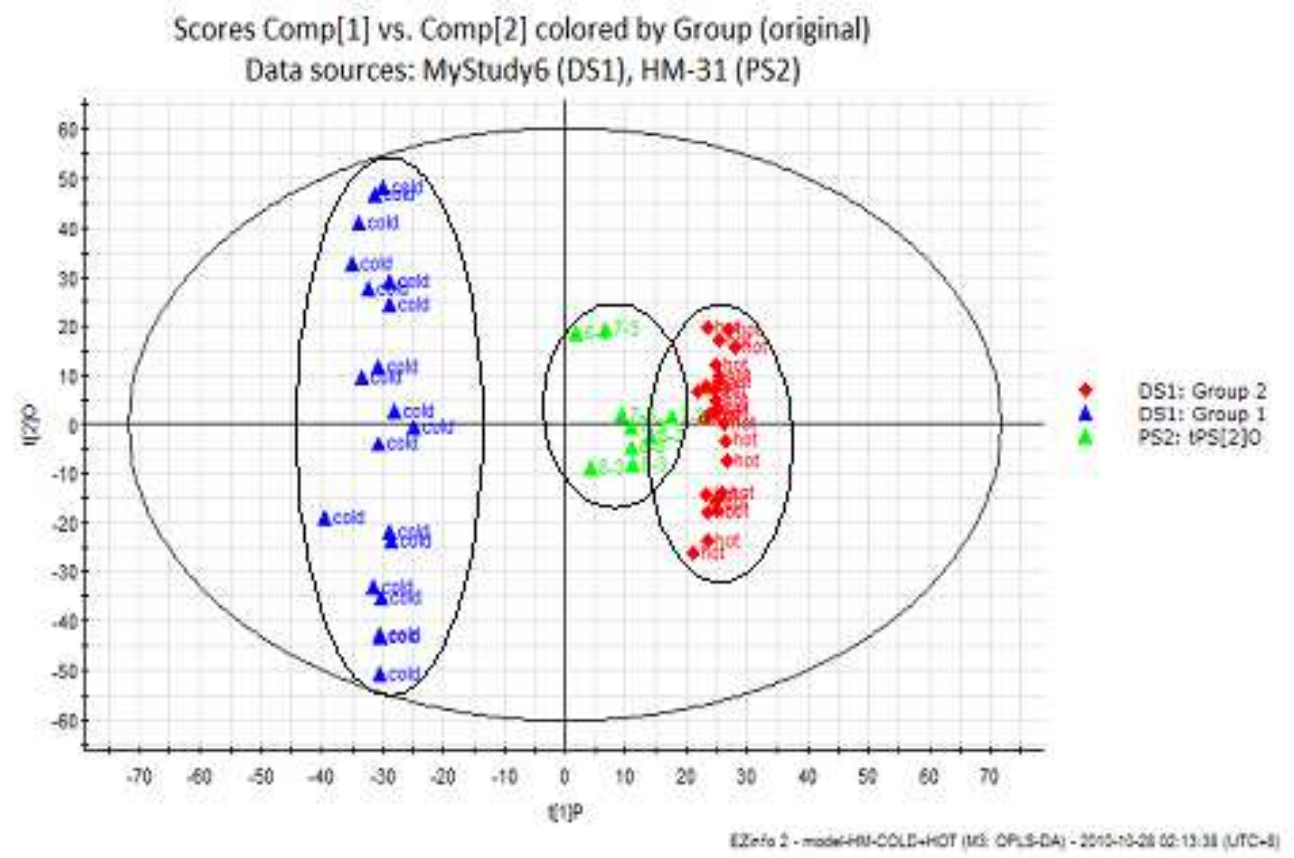

Fig. 5. Another hot medication-Flos Datura was used to verify the reliability of the forecasting model of OPLD-DA. $(\mathbf{\Delta})$ cold medication group, $(\bullet)$ hot medication group. $(\mathbf{\Delta})$ Flos Datura.

time, precise molecular mass and MS/MS data for the structural identification of biomarkers. The precise molecular mass was determined within measurement errors $(<5$ ppm) by Q-TOF, and meanwhile, the potential elemental composition and fractional isotope abundance of compounds were obtained. The presumed molecular formula was searched in METLIN Database and other databases to identify the possible chemical constitutions, and MS/MS data were screened to determine the potential structures of the ions. Taking two ions as examples, the identification procedure was as follows. Taking two ions as examples, the identification procedure was as follows. In the positive mode, the ion at $\mathrm{Rt}=5.46$ and $[\mathrm{M}+\mathrm{H}]^{+}=146$ has a high VIP value. This ion might contain an odd number of nitrogen atoms because its precise molecular weight was 145.0739, and its molecular formula was speculated as $\mathrm{C}_{6} \mathrm{H}_{11} \mathrm{NO}_{3}$ from the analysis of its elemental composition and fractional isotope abundance. The main fragment ions analyzed by MS/MS screening were $\mathrm{m} / \mathrm{z} 128$ and 101, which could be the $[\mathrm{M}+\mathrm{H}]^{+}$of lost $-\mathrm{NH}_{4}$, and $-\mathrm{C}_{2} \mathrm{H}_{7} \mathrm{~N}$, respectively. Finally, it was speculated as (S)-5-Amino-3-oxohexanoic acid, the ion at $\mathrm{Rt}=4.26$ and $[\mathrm{M}+\mathrm{H}]^{+}=162$ has a high VIP value, and the main fragment ions were $\mathrm{m} / \mathrm{z} 145,144$ and 117, which could be the $[\mathrm{M}+\mathrm{H}]^{+}$of lost $-\mathrm{NH}_{4},-\mathrm{NH}_{3}$ and $-\mathrm{NO}_{2}$, respectively. Finally, it was speculated as L-2Aminoadipic acid. 


\begin{tabular}{|c|c|c|c|c|c|c|c|c|}
\hline NO. & $\begin{array}{l}\text { VIP } \\
\text { values }\end{array}$ & Rt-M+ & Actual M & $\begin{array}{l}\text { Proposed } \\
\text { compound }\end{array}$ & Trend & $\begin{array}{l}\text { MS/ } \\
\text { MS }\end{array}$ & Losses & $\begin{array}{l}\text { Proposed } \\
\text { structure }\end{array}$ \\
\hline 1 & 2.70 & 5.25_134.0986 & 133.0375 & $\begin{array}{l}\text { Aspartic Acid } \\
\mathrm{C}_{4} \mathrm{H}_{7} \mathrm{NO}_{4}\end{array}$ & $\operatorname{Hot} \uparrow$ & $\begin{array}{l}118 \\
103\end{array}$ & $\begin{array}{l}\mathrm{O} \\
\mathrm{HNO}\end{array}$ & \\
\hline 2 & 3.00 & 3.64_190.041 & 189.0637 & $\begin{array}{l}\text { N-Acetyl-L- } \\
\text { glutamate } \\
\mathrm{C}_{7} \mathrm{H}_{11} \mathrm{NO}_{5}\end{array}$ & Cold $\downarrow$ & $\begin{array}{l}172 \\
146 \\
133\end{array}$ & $\begin{array}{l}\mathrm{CH}_{6} \\
\mathrm{C}_{2} \mathrm{H}_{4} \mathrm{O} \\
\mathrm{C}_{2} \mathrm{H}_{3} \mathrm{NO}\end{array}$ & \\
\hline 3 & 2.71 & 3.94_135.0962 & 134.0215 & $\begin{array}{l}\text { L-Apple acid } \\
\mathrm{C}_{4} \mathrm{H}_{6} \mathrm{O}_{5}\end{array}$ & Cold $\downarrow$ & 104 & $\mathrm{NO}$ & \\
\hline 4 & 2.13 & 4.73_233.0837 & 232.1212 & $\begin{array}{l}\text { Melatonin } \\
\mathrm{C}_{13} \mathrm{H}_{16} \mathrm{~N}_{2} \mathrm{O}_{2}\end{array}$ & Cold $\downarrow$ & $\begin{array}{l}191 \\
174\end{array}$ & $\begin{array}{l}\mathrm{C}_{2} \mathrm{H}_{2} \mathrm{O} \\
\mathrm{C}_{2} \mathrm{H}_{5} \mathrm{NO}\end{array}$ & \\
\hline 5 & 3.25 & 2.62_137.0683 & 136.0385 & $\begin{array}{l}\text { Hypoxanthine } \\
\mathrm{C}_{5} \mathrm{H}_{4} \mathrm{~N}_{4} \mathrm{O}\end{array}$ & Cold $\uparrow$ & 120 & $\mathrm{HO}$ & \\
\hline 6 & 5.84 & 2.7_180.0886 & 179.0582 & $\begin{array}{l}\text { Acetylisoniazi } \\
\text { d } \\
\mathrm{C}_{8} \mathrm{H}_{9} \mathrm{~N}_{3} \mathrm{O}_{2}\end{array}$ & Cold $\uparrow$ & 135 & $\mathrm{CHO}_{2}$ & \\
\hline 7 & 2.20 & $6.25 \_285.0773$ & 284.0757 & $\begin{array}{l}\text { Xanthosine } \\
\mathrm{C}_{10} \mathrm{H}_{12} \mathrm{~N}_{4} \mathrm{O}_{6}\end{array}$ & Cold $\uparrow$ & $\begin{array}{l}267 \\
228\end{array}$ & $\begin{array}{l}\mathrm{H}_{2} \mathrm{O} \\
\mathrm{CHN}_{2} \mathrm{O}\end{array}$ & \\
\hline 8 & 7.76 & 2.81_300.1645 & 299.0770 & $\begin{array}{l}\text { D-4'- } \\
\text { Phosphopanto } \\
\text { thenate } \\
\mathrm{C}_{9} \mathrm{H}_{18} \mathrm{NO}_{8} \mathrm{P}\end{array}$ & Cold $\uparrow$ & $\begin{array}{l}242 \\
187 \\
114\end{array}$ & $\begin{array}{l}\mathrm{C}_{2} \mathrm{H}_{2} \mathrm{O}_{2} \\
\mathrm{H}_{2} \mathrm{O}_{5} \mathrm{P} \\
\mathrm{C}_{3} \mathrm{H}_{9} \mathrm{NO}_{6} \\
\mathrm{P}\end{array}$ & \\
\hline 9 & 2.91 & 3.26_308.0929 & 307.0838 & $\begin{array}{l}\text { Glutathione } \\
\mathrm{C}_{10} \mathrm{H}_{17} \mathrm{~N}_{3} \mathrm{O}_{6} \mathrm{~S}\end{array}$ & Cold $\uparrow$ & $\begin{array}{l}262 \\
179 \\
147\end{array}$ & $\begin{array}{l}\mathrm{CH}_{2} \mathrm{~S} \\
\mathrm{C}_{5} \mathrm{H}_{7} \mathrm{NO}_{3} \\
\mathrm{C}_{5} \mathrm{H}_{7} \mathrm{NO}_{3} \\
\mathrm{~S}\end{array}$ & \\
\hline 10 & 2.25 & 3.04_133.0853 & 132.0535 & $\begin{array}{l}\text { L-Asparagine } \\
\mathrm{C}_{4} \mathrm{H}_{8} \mathrm{~N}_{2} \mathrm{O}_{3}\end{array}$ & Hot $\downarrow$ & 118 & $\mathrm{HN}$ & \\
\hline
\end{tabular}




\begin{tabular}{|c|c|c|c|c|c|c|c|c|}
\hline NO. & $\begin{array}{l}\text { VIP } \\
\text { values }\end{array}$ & Rt-M+ & Actual M & $\begin{array}{l}\text { Proposed } \\
\text { compound }\end{array}$ & Trend & $\begin{array}{l}\text { MS/ } \\
\text { MS }\end{array}$ & Losses & $\begin{array}{l}\text { Proposed } \\
\text { structure }\end{array}$ \\
\hline 11 & 5.02 & $4.14 \_146.0618$ & 145.0739 & $\begin{array}{l}\text { (S)-5-Amino- } \\
\text { 3-oxohexanoic } \\
\text { acid } \\
\mathrm{C}_{6} \mathrm{H}_{11} \mathrm{NO}_{3}\end{array}$ & Hot $\downarrow$ & $\begin{array}{l}128 \\
101\end{array}$ & $\begin{array}{l}\mathrm{H}_{4} \mathrm{~N} \\
\mathrm{C}_{2} \mathrm{H}_{7} \mathrm{~N}\end{array}$ & $\mathrm{H}_{3} \mathrm{C}^{-}$ \\
\hline 12 & 3.23 & 3.5_178.0469 & 177.046 & $\begin{array}{l}\text { N-Formyl-L- } \\
\text { methionine } \\
\mathrm{C}_{6} \mathrm{H}_{11} \mathrm{NO}_{3} \mathrm{~S}\end{array}$ & Hot $\downarrow$ & $\begin{array}{l}148 \\
132 \\
104\end{array}$ & $\begin{array}{l}\mathrm{CH}_{2} \mathrm{O} \\
\mathrm{CH}_{2} \mathrm{~S} \\
\mathrm{C}_{2} \mathrm{H}_{2} \mathrm{OS}\end{array}$ & \\
\hline 13 & 4.31 & 9.57_301.1442 & 300.2089 & $\begin{array}{l}\text { Vitamin } \mathrm{A} \\
\text { acid } \\
\mathrm{C}_{20} \mathrm{H}_{28} \mathrm{O}_{2}\end{array}$ & Hot $\downarrow$ & $\begin{array}{l}284 \\
175\end{array}$ & $\begin{array}{l}\mathrm{HO} \\
\mathrm{C}_{9} \mathrm{H}_{18}\end{array}$ & $\mathrm{CH}_{3}$ \\
\hline 14 & 5.13 & $4.1 \_289.1075$ & 288.0246 & $\begin{array}{l}\text { 2-Dehydro-3- } \\
\text { deoxy-D- } \\
\text { arabino- } \\
\text { heptonate 7- } \\
\text { phosphate } \\
(\mathrm{DAHP}) \\
\mathrm{C}_{7} \mathrm{H}_{13} \mathrm{O}_{10} \mathrm{P}\end{array}$ & Hot $\uparrow$ & $\begin{array}{l}242 \\
158\end{array}$ & $\begin{array}{l}\mathrm{CH}_{3} \mathrm{O}_{2} \\
\mathrm{H}_{4} \mathrm{O}_{6} \mathrm{P}\end{array}$ & \\
\hline 15 & 2.78 & 4.05_151.0389 & 150.1045 & $\begin{array}{l}\text { Myrtenal } \\
\mathrm{C}_{10} \mathrm{H}_{14} \mathrm{O}\end{array}$ & Hot $\uparrow$ & $\begin{array}{l}123 \\
108\end{array}$ & $\begin{array}{l}\mathrm{C}_{2} \mathrm{H}_{4} \\
\mathrm{C}_{3} \mathrm{H}_{7}\end{array}$ & \\
\hline 16 & 12.73 & 4.26_162.0519 & 161.0688 & $\begin{array}{l}\text { L-2- } \\
\text { Aminoadipic } \\
\text { acid } \\
\mathrm{C}_{6} \mathrm{H}_{11} \mathrm{NO}_{4}\end{array}$ & $\operatorname{Hot} \uparrow$ & $\begin{array}{l}145 \\
144 \\
117\end{array}$ & $\begin{array}{l}\mathrm{NH}_{3} \\
\mathrm{NH}_{4} \\
\mathrm{NO}_{2}\end{array}$ & \\
\hline 17 & 2.04 & 3.89_319.1289 & 318.2195 & $\begin{array}{l}\text { Leukotriene } \\
\text { A4 } \\
\mathrm{C}_{20} \mathrm{H}_{30} \mathrm{O}_{3}\end{array}$ & $\operatorname{Hot} \uparrow$ & $\begin{array}{l}273 \\
259 \\
162\end{array}$ & $\begin{array}{l}\mathrm{C}_{3} \mathrm{H}_{10} \\
\mathrm{C}_{4} \mathrm{H}_{12} \\
\mathrm{C}_{8} \mathrm{H}_{13} \mathrm{O}_{3}\end{array}$ & \\
\hline 18 & 20.75 & 4.48_164.0674 & 163.0633 & $\begin{array}{l}\text { 3- } \\
\text { Methyldioxyi } \\
\text { ndole } \\
\mathrm{C}_{9} \mathrm{H}_{9} \mathrm{NO}_{2}\end{array}$ & Cold $\downarrow$ & $\begin{array}{l}146 \\
122\end{array}$ & $\begin{array}{l}-\mathrm{H} 2 \mathrm{O} \\
-\mathrm{CNO}\end{array}$ & \\
\hline
\end{tabular}

Table 2. Potential biomarkers identified in positive ESI mode.

\section{Discussion}

Metabonomics aims to assess metabolic changes in a comprehensive and global manner in order to infer biological functions and provide the detailed biochemical responses of cellular 
systems [19]. In this study the successful discrimination and classification of the four properties in TCM was made. Multivariate statistical analysis was used to find the most characteristic markers in complex records. This demonstrates the potential of UPLC-QTOFMS combined with metabonomics to determine the four properties of TCM. It should be noted that for this particular purpose, the molecular identification of these marker compounds is not necessary, some different chemometric tools, such as PCA and OPLS-DA have been proposed as powerful tools for the four properties classification [20]. However, the benefit of obtaining MS/MS accurate mass spectra of marker compounds (metabolites) were shown to provide a high level of confidence for the identification process. Even with this data, the identification of true unknowns is a rather difficult task. Biological Interpretation of several biomarkers: L-Apple acid is a tart-tasting organic dicarboxylic acid that plays a role in many sour or tart foods. In its ionized form it is malate, an intermediate of the TCA cycle along with fumarate. It can also be formed from pyruvate as one of the anaplerotic reactions. It is a key intermediate in energy metabolism and the change indicated that energy metabolism was perturbation by Chinese herbs.

\section{Conclusion}

The four properties, the essence and important component of TCM theories, the high generalization on the basic property and typical characteristics of TCM, are a significant theoretical foundation for the clinical use of Chinese medicine. In this study, UPLC-QTOFMS techniques coupled to metabonomics methods were used to prove the existence of the four properties, to illustrate its multi-component, multi-target, multi-channel and the complex mechanism. All the work are aimed at guiding researchers to carry out new drug develop work with the theory of Chinese medicine, simultaneously, eliminating too much emphasis the effective chemical composition while ignore the many other ones, result in the loss of characteristics of Chinese herbs and even highlights the toxicity and side effects. This theory also could apply to explore Western medicine properties to effectively guide the clinical application. Considering the encouraging results obtained in this study, the application of metabonomics for authentication and other purposes in TCM seems to be very promising approach [22].

\section{Acknowledgements}

Our work was supported by the Major State Basic Research Development Program of China (973 Program 2006CB504708) the National Natural Science Foundation of China (No. 30371736, 30672633) and Special Fund Project of National Excellent Doctoral Dissertation of China (200980).

\section{References}

[1] C. Auffray, Z. Chen, L. Hood, Genome Med.1 (2009) 1-2.

[2] T. Ma, C.g. Tan, H. Zhang, M.Q. Wang, W. J. Ding, S. Li, Mol. BioSyst. 6 (2010) 613-619.

[3] A.P. Lu, H.W. Jia, C. Xiao, Q.P. Lu.. World J Gastroenterol 13 (2004) 1854-1856.

[4] D. Normile. Science 299(2003) 188-190.

[5] J. Lindon, J. Nicholson, E. Holmes, Elsevier, Amsterdam, (2007) 279-287. 
[6] J. Chen,W.Z.Wang, S. Lv, P. Y. Yin, X. J. Zhao, X. Lu, F. X. Zhang, G.W. Xu, Analytica Chimica Acta 650 (2009) 3-9.

[7] D.M. Drexler, J.H.M. Feyen, M. Sanders, Drug Discov. Today Technol. 1 (2004) 17-23.

[8] J.C. Lindon, E. Holmes, M.E. Bollard, E.G. Stanley, J.K. Nicholson, Biomarkers 9 (2004) 131.

[9] D.G. Robertson, M.D. Reily, J.D. Baker, J. Proteome Res. 6 (2007) 526-539.

[10] I.D. Wilson, R. Plumb, J. Granger, H.Major, R.Williams, E.M. Lenz, J. Chromatogr. B 817 (2005) 67-76.

[11] E. Holmes, P.J.D. Foxall, M. Spraul, R.D. Farrant, J.K. Nicholson, J.C. Lindon, J. Pharm. Biomed. Anal. 15 (1997) 1647-1659.

[12] C. Wang, H.W. Kong, Y.F. Guan, J. Yang, J.R. Gu, S.L. Yang, G.W. Xu, Anal. Chem. 77 (2005) 4108-4116.

[13] F. Benavente, R. van der Heijden, U.R. Tjaden, J. van der Greef, T. Hankemeier, Electrophoresis 27 (2006) 4570-4584.

[14] J. Zhang, L. J. Yan, W. G. Chen, L. Lin, X. Y. Song, X. M. Yan,W. Hang, B. 1. Huang, Analytica Chimica Acta 650 (2009) 16-22.

[15] R.S. Plumb, J.H. Granger, C.L. Stumpf, K.A. Johnson, B.W. Smith, S. Gaulitz, I.D. Wilson, J. Castro-Perez, Analyst 130 (2005) 844-849.

[16] J.H. Granger, R. Williams, E.M. Lenz, R.S. Plumb, C.L. Stumpf, I.D. Wilson, Rapid Commun. Mass Spectrom. 21 (2007) 2039-2045.

[17] R. Madsen, T. Lundstedt, J. Trygg, Analytica Chimica Acta 659 (2010) 23-33.

[18] P. Wang, H. Sun, H.T. Lv, W. J. Sun, Y. Yuan, Y. Han, D. W. Wang, A. H. Zhang, X. J. Wang, J. Pharm. Biomed. Anal. 53 (2010) 631-645.

[19] O. Fiehn;, B. Kristal, B. van Ommen, L. W. Sumner, S. A. Sansone, C. Taylor, N. Hardy, R. Kaddurah-Daouk, OMICS, 10 (2006), 158-63.

[20]L. Rafael, U. S. Mireia, J.Olga, M.Maria, A. L. Cristina, Journal of Proteome Research, 8(2009), 5060-5068.

[21] S.Wiklund, E. Johansson, L. Sjostrom, E. J. Mellerowicz, U. Edlund, J. P. Shockcor, J. Gottfries, T. Moritz, J. Trygg, Anal. Chem., 80 (2008), 115-22.

[22] F. Y. Du, Y. Bai, Y. Bai, H.W. Liu, Anal. Chem. 82 (2010), 9374-9383. 


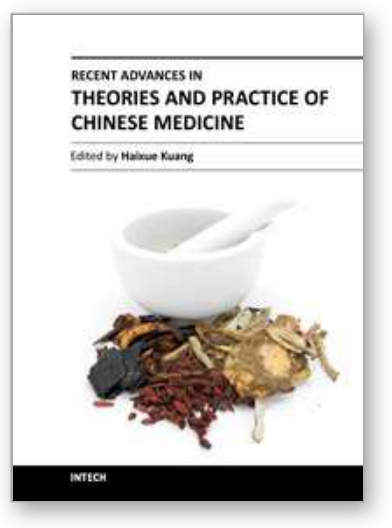

\author{
Recent Advances in Theories and Practice of Chinese Medicine \\ Edited by Prof. Haixue Kuang
}

ISBN 978-953-307-903-5

Hard cover, 504 pages

Publisher InTech

Published online 18, January, 2012

Published in print edition January, 2012

During the recent years, traditional Chinese medicine (TCM) has attracted the attention of researchers all over the world. It is looked upon not only as a bright pearl, but also a treasure house of ancient Chinese culture. Nowadays, TCM has become a subject area with high potential and the possibility for original innovation. This book titled Recent Advances in Theories and Practice of Chinese Medicine provides an authoritative and cutting-edge insight into TCM research, including its basic theories, diagnostic approach, current clinical applications, latest advances, and more. It discusses many often neglected important issues, such as the theory of TCM property, and how to carry out TCM research in the direction of TCM property theory using modern scientific technology. The authors of this book comprise an international group of recognized researchers who possess abundant clinical knowledge and research background due to their years of practicing TCM. Hopefully, this book will help our readers gain a deeper understanding of the unique characteristics of Chinese medicine.

\title{
How to reference
}

In order to correctly reference this scholarly work, feel free to copy and paste the following:

Haixue Kuang, Yanyan Wang, Qiuhong Wang, Bingyou Yang and Yonggang Xia (2012). Metabonomics Research of the Four Properties in Traditional Chinese Medicine Based on UPLC-QTOF-MS System, Recent Advances in Theories and Practice of Chinese Medicine, Prof. Haixue Kuang (Ed.), ISBN: 978-953-307-903-5, InTech, Available from: http://www.intechopen.com/books/recent-advances-in-theories-and-practice-ofchinese-medicine/metabonomics-research-of-the-four-properties-in-traditional-chinese-medicine-based-onuplc-qtof-ms-s

\section{INTECH}

open science | open minds

\section{InTech Europe}

University Campus STeP Ri

Slavka Krautzeka 83/A

51000 Rijeka, Croatia

Phone: +385 (51) 770447

Fax: +385 (51) 686166

www.intechopen.com

\section{InTech China}

Unit 405, Office Block, Hotel Equatorial Shanghai

No.65, Yan An Road (West), Shanghai, 200040, China

中国上海市延安西路65号上海国际贵都大饭店办公楼 405 单元

Phone: +86-21-62489820

Fax: +86-21-62489821 
(C) 2012 The Author(s). Licensee IntechOpen. This is an open access article distributed under the terms of the Creative Commons Attribution 3.0 License, which permits unrestricted use, distribution, and reproduction in any medium, provided the original work is properly cited. 